$24{ }^{\dagger}$ These authors contributed equally to the work

$25 * *$ Data used in preparation of this article were obtained from the Alzheimer's Disease

\section{Gray matter volume and estimated brain age gap are not associated with}

\section{sleep-disordered breathing in subjects from the ADNI cohort}

Bahram Mohajer $^{\mathrm{a}, \mathrm{b}}$, Nooshin Abbasi ${ }^{\mathrm{c} \dagger}$, Esmaeil Mohammadi ${ }^{\mathrm{a}, \mathrm{b} \dagger}$, Habibolah Khazaie ${ }^{\mathrm{d}}$, Ricardo S.

Osorio $^{\mathrm{e}, \mathrm{f}}$, Ivana Rosenzweig ${ }^{\mathrm{g}, \mathrm{h}}$, Claudia R. Eickhoff ${ }^{\mathrm{i}, \mathrm{k}}$, Mojtaba Zarei ${ }^{\mathrm{a}}$, Masoud Tahmasian ${ }^{\mathrm{a} *}$, Simon B. Eickhoff ${ }^{\mathrm{i}, \mathrm{j}}$ for the Alzheimer's Disease Neuroimaging Initiative**

a) Institute of Medical Science and Technology, Shahid Beheshti University, Tehran, Iran

b) Non-Communicable Diseases Research Center, Endocrinology and Metabolism Population Sciences Institute, Tehran University of Medical Sciences, Tehran, Iran

c) McConnell Brain Imaging Centre, Montreal Neurological Institute, McGill University, Montreal, QC, Canada

d) Sleep Disorders Research Center, Kermanshah University of Medical Sciences, Kermanshah, Iran

e) Department of Psychiatry, Center for Brain Health, NYU Langone Medical Center, New York, NY, USA

f) Nathan S. Kline Institute for Psychiatric Research, Orangeburg, New York, NY, USA

g) Sleep Disorders Centre, Guy's and St Thomas' Hospital, GSTT NHS, London, UK

h) Sleep and Brain Plasticity Centre, Department of Neuroimaging, IOPPN, King's College London, London, UK

i) Institute of Neuroscience and Medicine (INM-1; INM-7), Research Center Jülich, Jülich, Germany

j) Institute of Systems Neuroscience, Medical Faculty, Heinrich-Heine University, Düsseldorf, Germany

k) Institute of Clinical Neuroscience and Medical Psychology, Heinrich Heine University, Düsseldorf, Germany

Neuroimaging Initiative (ADNI) database (adni.loni.usc.edu). As such, the investigators within 
27 the ADNI contributed to the design and implementation of ADNI and/or provided data but did

28 not participate in analysis or writing of this report. A complete listing of ADNI investigators can

29 be found at:

30 http://adni.loni.usc.edu/wp-content/uploads/how to apply/ADNI Acknowledgement List.pdf

31

32 * Corresponding author: Masoud Tahmasian M.D., Ph.D., Institute of Medical Science and

33 Technology, Shahid Beheshti University, Daneshjou Boulevard, Velenjak, P.O. Box

34 1983969411, Tehran, Iran. Telephone: +98-21-29905803; Fax: +98-21-29902650;

35 Email: $\underline{\text { m_tahmasian@sbu.ac.ir }}$ 


\section{Abstract:}

37 Alzheimer's disease (AD) and sleep-disordered breathing (SDB) are prevalent conditions with

38 rising burden. It is suggested that SDB may contribute to cognitive decline and advanced aging.

39 Here, we assessed the link between self-reported SDB and gray matter volume in patients with

$40 \mathrm{AD}$, mild cognitive impairment (MCI) and healthy controls (HC). We further investigated

41 whether SDB was associated with advanced brain aging. We included a total of 330 participants,

42 divided based on self-reported history of SDB, and matched across diagnoses for age, sex and

43 presence of the ApoE4 allele, from the Alzheimer's Disease Neuroimaging Initiative. Gray-

44 matter volume was measured using voxel-wise morphometry and differences reflecting SDB,

45 cognitive status, and their interaction were evaluated. Further, using an age-prediction model

46 fitted on gray-matter data of external datasets, we predicted study participants' age from their

47 structural scans. Cognitive decline (MCI/AD diagnosis) and advanced age were associated with

48 lower gray matter volume in various regions, particularly in the bilateral temporal lobes.

49 BrainAGE was well predicted from the morphological data in $\mathrm{HC}$ and, as expected, elevated in

$50 \mathrm{MCI}$ and particularly in AD. However, there was neither a significant difference between

51 regional gray matter volume in any diagnostic group related to the SDB status nor an SDB-by-

52 cognitive status interaction. Also, we found neither a significant difference in BrainAGE gap

53 (estimated - chronological age) related to SDB nor an SDB-by-cognitive status interaction. In

54 summary, contrary to our expectations, we were not able to find a general nor a diagnostic

55 specific effect on either gray-matter volumetric or brain aging.

56 Statement of significance: Dementia syndromes including Alzheimer's disease (AD), are a

57 major global concern, and unraveling modifiable predisposing risk factors is indispensable.

58 Sleep-disordered breathing (SDB) and its most prevalent form, obstructive sleep apnea, are 
59 suggested as modifiable risk factors of $\mathrm{AD}$, but their contribution to $\mathrm{AD}$ hallmarks, like brain

60 atrophy and advanced brain aging, is not clear to this day. While self-reported SDB is suggested

61 to propagate aging process and cognitive decline to AD in clinical studies, here, we demonstrated

62 that, SDB might not necessarily associate to brain atrophy and the advanced brain aging assessed

63 by morphological data, in $\mathrm{AD}$ progession. However, multimodal longitudinal studies with

64 polysomnographic assessment of SDB are needed to confirm such fundings.

65 Keywords: Sleep-disordered breathing; Obstructive Sleep Apnea; Alzheimer's disease; Mild

66 cognitive impairment; Age prediction; BrainAGE; Gray matter volume; Alzheimer's Disease

67 Neuroimaging Initiative

68 


\section{Introduction:}

70

71 prevalence of 712 cases per 100,000 population in 2016, affecting 40-50 million people

72 worldwide ${ }^{1}$. Considering that the numbers of $\mathrm{AD}$ patients have been more than doubled during

73 past three decades ${ }^{1}$, it is critical to unravel the predisposing risk factors ${ }^{2}$. These include

74 advanced aging of the world population, but also modifiable risk factors such as cardiovascular

75 disease, diabetes ${ }^{2}$, obesity ${ }^{3}$, and potentially sleep-disordered breathing (SDB) ${ }^{4}$. SDB ranges from

76 partial (episodical) to complete airway obstruction leading to intermittent hypoxia, sleep

77 fragmentation and intrathoracic pressure swings ${ }^{5}$. A bidirectional relationship has been proposed

78 for SDB, including its most common form, obstructive sleep apnea (OSA), and AD. In

79 particular, it has been suggested that patients with OSA are more likely to develop mild cognitive

80 impairment (MCI) or dementia ${ }^{6,7}$. Moreover, a meta-analysis demonstrated that prevalence of

81 OSA is five times higher in patients with $\mathrm{AD}$ than cognitively unimpaired individuals of the

82 same age ${ }^{4}$.

Gray matter atrophy is a prime feature of pathologic brain aging ${ }^{8}$ and a well-known

84 finding in $\mathrm{AD}$, starting primarily in the medial temporal region and then globally affecting the

85 brain as the disease progresses ${ }^{9-11}$. Morphometric analysis of the structural magnetic resonance

86 images (MRI) has shown to reliably reveal this effect ${ }^{12}$. While some studies have shown gray

87 matter atrophy in brain regions like the hippocampus, a key region involved in $\mathrm{AD}$, to be

88 associated with SDB in non-demented subjects ${ }^{13-16}$, others have shown either null results ${ }^{17}$ or

89 paradoxical hypertrophy or thickening of gray matter in SDB ${ }^{18-24}$. Discrepancy between these

90 findings is attributed to variations in cognitive status of participants, definitions of SDB severity,

91 and method of gray matter volume assessment ${ }^{14-16,18-24}$. Thus, it remains unclear, whether SDB 
92 may result in brain atrophy similar to the volume changes in $\mathrm{AD}$ and hence contribute to its

93 pathophysiology.

94 Aside from regional atrophy of the medial temporal lobe, $\mathrm{AD}$ is associated with advanced

95 multivariate patterns of brain aging. In particular, it has been shown that individual subjects' age

96 can be predicted from gray matter morphometry in the cognitively normal population using

97 machine-learning approaches ${ }^{25}$. That is, models trained to predict individuals ages based on

98 larger cohorts of reference scans allow to estimate the age of a new person with a mean accuracy

99 of 4-5 years ${ }^{26}$, while neurodegenerative disorders show a reliable pattern of advanced aging, i.e.,

100 a positive BrainAGE score (difference between age predicted based on the morphometric pattern

101 and chronological age $)^{25,27-29}$. Whether accelerated brain aging as seen in AD and to a lesser

102 degree MCI is also present in potentially related conditions such as SDB is still an open question.

103 The aim of the current study is to shed further light on the potential relationship between

104 brain atrophy patterns in SDB and AD at the regional and global level, answering two questions.

105 1) Do patients with SDB show grey matter atrophy across or in interaction with cognitive status

106 (healthy control (HC), MCI, AD)? 2) Do patients with SDB show advanced brain aging across or

107 in interaction with cognitive status (HC, MCI, AD)? To this end, we used data from the

108 Alzheimer's Disease Neuroimaging Initiative (ADNI), and established the validity of our

109 methods by replicating previous findings for both aims in MCI and AD, and then assessed gray

110 matter volume and BrainAGE differences between SBD+ and SBD-, including interactions with

111 cognitive status. 


\section{Methods:}

\section{Participants}

115 database (adni.loni.usc.edu $)^{30}$ based on their cognitive status and the medical history regarding

$116 \mathrm{SDB}^{7}$. Diagnoses of MCI and AD were based on the ADNI criteria. Subjects with self-reported

117 "sleep apnea" or "obstructive sleep apnea" or "OSA" symptoms or receiving treatment with

118 "Continuous Positive Airway Pressure" (or "CPAP") or "Bilevel Positive Airway Pressure" (or

119 "BiPAP”/“BPAP”) were labeled as "SDB+". wo independent physicians reviewed medical

120 history to confirm diagnosis and grouping the subjects. Demographic and clinical variables were

121 extracted for all individuals, missing covariate data were assessed and imputation was used for 5

122 participants with missing data-points. Using 1:1 propensity score matching method, we

123 assembled 6 distinct sub-groups according to their cognitive (HC, MCI, AD) and SDB (SDB+

124 and SDB-) status. Covariates included in the matching were age, sex, years of education, body-

125 mass index, cognitive status (AD/MCI/HC), presence of the Apolipoprotein E4 (ApoE4) allele,

126 history of SDB treatment (only when matching between SDB+ subjects), T1 imaging protocol

127 and, field strength (Table 1). Only subjects that passed the quality assessment tools of the CAT

128 toolbox, including weighted image quality rating based on basic image properties and noise and

129 geometric distortions, as well as checking homogeneity through the sample, were considered.

\section{Imaging acquisition and preprocessing}

Participants had undergone a standardized protocol for high-resolution MRI T1 scans of

132 the brain as previously described ${ }^{31}$. T1 imaging acquisition parameters were: $\mathrm{TR}=2400 \mathrm{~ms}$,

133 minimum full TE, TI=1000 ms, flip angle $=8^{\circ}, 24 \mathrm{~cm}$ field of view, acquisition matrix of 192

$134 \times 192 \times 166$ and with $1.25 \times 1.25 \times 1.2 \mathrm{~mm} 3$ slice size. We used Computational Anatomy Toolbox 
135 (CAT) v12 ${ }^{32}$ and SPM12 (www.fil.ac.uk/spm) to perform voxel-based morphometry (VBM).

136 This included correcting the bias-field distortions and noise removal, skull stripping,

137 normalization to standard space and brain tissue segmentation into grey matter, white matter, and

138 cerebrospinal fluid. Grey matter segments were modulated to represent actual gray matter

139 volume. We then performed a biologically informed compression of the VBM data to 637 gray

140 matter parcels based existing in-vivo brain parcellation ${ }^{33},{ }^{34}$. Thus, grey matter volume of each

141 participant was represented by 637 features each representing an individual parcel volume of that

142 participant. All consecutive analyses were performed on this data.

\section{Statistical analysis of gray matter volume}

Statistical analysis of gray matter volume of parcels included three consecutive parts,

146 similar to approach used by Bludau et. $\mathrm{al}^{35}$; generating reference statistics, permuted statistics,

147 and a family-wise error (FWE) correction for multiple comparisons. Here we used an n-way

148 analysis of variance, to test effect of age, cognitive status (AD/MCI/HC), SDB status and SDB-

149 by-cognitive status interaction, separately as independent variables (factors), on gray matter

150 volume of each parcel as dependent variable. The F values (per parcel) of this ANOVA were

151 considered as the reference statistics. In the subsequent permutation statistics for each factor, we

152 randomly shuffled the labels for that factor 10,000 times, replicated the analysis and recorded the

153 F-values to build a null-distribution. The comparison of the reference statistic with this

154 distribution then allows non-parametric inference per parcel and factor, yielding uncorrected p-

155 values. Importantly, however, we also recorded, per replication of the permutation, the highest

156 statistics in the random data across the entire set, i.e., 637 brain regions, building a null- 
157 distribution for family-wise error correction. The threshold corresponding to $\mathrm{p}_{\mathrm{FWE}}<0.05$ was

158 then provided by the (set-wise maximum) value exceeded only in $5 \%$ of the replications.

\section{Age prediction}

Brain age was estimated from the atlas-based representations of individual brain anatomy

161 using a support vector machine (SVM) ensemble model. An independent (reference) large

162 dataset consisting of 2089 (Figure 1A.1) subjects (between 55 and 85 years old) was compiled

163 from several large public and private datasets including 1000 Brains $^{36}$, Cambridge Centre for

164 Ageing and Neuroscience or Cam-CAN ${ }^{37}$, OpenfMRI ${ }^{38}$, Dallas Lifespan Brain Study or DLBS,

165 Consortium for Reliability and Reproducibility or CoRR ${ }^{39}$, IXI, and Enhanced Nathan Kline

166 Institute-Rockland Sample or eNKI-RS ${ }^{40}$. Given the imbalance between age brackets, sites, and

167 sex, we performed a stratified subsampling, choosing the same number of men and women, as

168 well as similar numbers across age-brackets and a maximum of 30 subjects per age-bracket and

169 sex per site. The actual subjects sampled in each replication from the overall database were

170 drawn from the pool independently at random without replacement. Each of these sampled sets

171 was then used to fit an individual SVM providing a weak learner for the ensemble which was

172 applied to the test-data, i.e., the ADNI sample. The process was repeated 10,000 times, yielding

17310,000 age predictions based on models trained on (different) balanced subsamples of the multi-

174 cohort reference data. These predictions were then averaged ("bagging") to yield the final age

175 prediction based on the 637-parcel representation of the VBM data ${ }^{41}$. Each subjects BrainAGE

176 score was finally calculated as bagged predicted age minus chronological age for each subject

177 (Figure 1). 


\section{$178 \quad$ Results}

179 Both SDB+ and SDB- groups comprised of $24 \mathrm{AD}, 111 \mathrm{MCI}$, and $30 \mathrm{HC}$ participants,

180 respectively. As enforced through the matching, there was no statistically significant difference

181 in demographic variables, cognitive status, and presence of the ApoE4 allele between SDB

182 groups. Table 1 summarizes the characteristics of all study groups.

\section{Effects on grey matter volume}

As noted in the methods, association of parcel-wise gray matter volume with age, cognitive status, SDB status, and SDB-by-cognitive status interaction was assessed using nonparametric inference with FWE correction for multiple comparisons. There were strong $\left(\mathrm{P}_{\mathrm{FWE}}\right.$

$187<0.001)$ and widespread negative associations of regional grey matter volume with age, in

188 particular in the bilateral temporal lobes, bilateral prefrontal, middle and superior frontal areas,

189 bilateral medial and lateral occipital areas, cerebellum and thalamus, caudate and putamen in the

190 subcortical gray matter (Figure 2A). The cognitive status was significantly associated with 191 reduced gray matter volume in many bilateral parcels with dominancy in the left hemisphere 192 ( $\left.\mathrm{P}_{\mathrm{FWE}}<0.001\right)$. Bilateral temporal lobes including fusiform gyri, medial temporal lobes and 193 hippocampal formations, and inferior and middle temporal lobes had significantly lower volume 194 in participants with MCI and particularly AD. Moreover, reduced gray matter volume was seen 195 in bilateral insula, middle frontal, and cingulate cortices, as well as left superior frontal cortex 196 (Figure 2B). In turn, when testing for effects of SDB status and SDB-by-cognitive status 197 interaction, we found no significant regions anywhere in the brain (all $\left.\mathrm{P}_{\mathrm{FWE}}>0.05\right)$.

\section{Effects on estimated brain age}


The mean absolute error between predicted and chronological age in the HC group was

2003.59 years, indicative of the very good performance of the ensemble prediction model. We then

201 calculated the BrainAGE score as the per-subject difference between predicted and chronological

202 age and tested for its association with cognitive status, SDB status, and the SDB-by-cognitive

203 status interaction. As it is shown in Figure 3, participants with MCI and in particular AD showed

204 an advanced brain age (on average 4.0 and 9.1 years, respectively), in line with previous studies.

205 However, there was no significant effect on BrainAGE scores associated with SDB status, nor

206 was there a positive SDB-by-cognitive status interaction suggesting that SDB may not lead to

207 advanced brain aging (Figure 3C). 


\section{Discussion}

Our findings confirmed previously reported gray matter atrophy and accelerated

210 biological brain aging in patients with MCI and $\mathrm{AD}$, corroborating the robustness and validity of

211 our analytical approach. Importantly, we were not able to demonstrate any effect of SDB,

212 independently or in interaction with cognitive status, on either regional grey matter volume or

213 brain aging score. Several limitations however, may compromise the interpretation of our results.

214 Sample sizes of SDB+ subjects in the HC and AD groups were small. Moreover, the groups were

215 heterogeneous in terms of clinical characteristics and imaging specifications. We used

216 propensity-score matching and stratified subsampling of external datasets to minimize the effects

217 of heterogeneity. As previously mentioned on publications using the ADNI database $e^{7,42}$, the self-

218 reported measure of SDB can be influenced by both the recall bias of cognitively impaired

219 subjects as well as by a high prevalence of undiagnosed OSA in the general population, therefore

220 increasing the probability of false negative cases in the SDB-groups ${ }^{7}$. Moreover, assessment of

221 the severity of SDB and disease duration were not available.

\section{Grey matter volume alterations in AD and SDB}

223 One of the main characteristics of MCI and AD is generalized gray matter loss in the

224 brain, which mostly starts in the medial temporal lobe and multimodal association areas ${ }^{8-10}$.

225 Neuroimaging meta-analyses have demonstrated atrophy in the medial temporal lobe, limbic

226 regions (left parahippocampl gyrus, left posterior cingulate gyrus, amygdala and uncus),

227 thalamus, temporal, parietal, frontal and cingulate cortices ${ }^{43,44}$. A similar but milder distribution

228 of gray matter atrophy is evident in brain of patients with $\mathrm{MCI}^{43,45}$. In accordance with the

229 previous brain volumetric studies, we found diffuse gray matter loss in MCI and AD. The 
230 atrophy was mainly located in the bilateral temporal lobe and medial temporal areas with higher

231 intensity in $\mathrm{AD}$ compared to MCI.

232 Assessing the volumetric changes due to SDB, we did not observe any significant 233 alteration in gray matter volume, neither in HC subjects, nor in patients with MCI or AD.

234 Furthermore, self-reported SDB interaction with cognitive status (i.e. HC, MCI or AD) showed

235 no associations with gray matter volume. Historically, there has been an inability to replicate

236 results among the brain imaging studies of SDB in non-demented populations. While several

237 studies have reported gray matter atrophy in the insula, amygdala, middle and lateral temporal

238 regions, and cerebellum in non-demented populations with $\mathrm{SDB}^{13-16,46-48}$, others have either

239 shown no associations ${ }^{17,49}$ or paradoxical enhancement in the gray matter volume of regions like

240 the motor cortices, prefrontal cortex, thalamus, putamen, and the hippocampus ${ }^{20-24,47}$. In

241 addition, there is a general lack of longitudinal studies, which would enable the study of non-

242 linear associations between SDB and cortical atrophy as suggested by these cross-sectional

243 findings. Despite these important gaps in the literature, three meta-analyses have concluded that

244 OSA is associated with gray matter atrophy in a few selected regions including the cingulate,

245 amygdala, hippocampus, right central insula, right middle temporal gyrus, right premotor cortex, 246 and cerebellum ${ }^{13,50,51}$.

247 The observed null association between SDB and gray matter volume should however be 248 interpreted with caution. First, it has been suggested that aging may have partially protective 249 mechanisms against SDB, such as reduced production of oxidative stress after apneas and 250 decreased blood pressure and heart rate responses after arousals ${ }^{23}$. The average old age of ADNI

251 subjects ( 75 years-old) could therefore explain this non-significant association between SDB

252 and brain morphometry. Despite numerous studies and meta-analyses focused on the changes in 
253 gray matter in middle-aged patients with OSA, there are few studies on gray matter changes in

254 older adults with SDB and neither have found any decreases in thickness or volume in cortical

255 gray matter ${ }^{52-54}$. Second, it is possible that SDB-related brain damage impacts more selectively

256 brain function ${ }^{55}$ or amyloid burden ${ }^{17}$ than gray matter volume alone, or that differential diagnosis

257 between SDB-related and age-related brain atrophy is difficult in single-point observational

258 studies, particulary in those cases in which groups are matched by age and cognitive status.

259 Third, this could also be a sign of: a) survival bias, as most SDB+ may have transitioned to AD

260 and only those with very low cortical atrophy or high in cognitive reserve at disease onset would

261 remain as $\mathrm{HC}$ or MCI at cross-section; or, b) selection bias due to matching by the ApoE4 allele,

262 as it has been reported that the ApoE4 allele interacts with brain aging scores measured by the

263 BrainAGE method, revealing potential neuronal compensation in healthy ApoE4+ adults ${ }^{73}$,

264 which could also result in null findings. Fourth, we did not account for other comorbidities and

265 possible confounders alongside age or presence of the ApoE4 allele in the prediction models ${ }^{72}$.

266 Finally, previous MRI studies mostly recruited patients with PSG-diagnosed OSA from sleep

267 clinics, which might be a different population from those recruited in memory clinics with self-

268 reported assessment of SDB based on clinical interview.

269 We were also not able to demonstrate any interaction between SDB and MCI or AD with

270 brain atrophy. This is indicative that despite the frequent clinical co-occurrence of SDB and AD,

271 there may be no synergy between them in accelerating gray matter atrophy. Recent investigations

272 using cerebrospinal fluid and PET imaging suggest an interplay between amyloid

273 production/clearance and $\mathrm{SDB}^{17,42,56-58}$. These include an impairment in the cerebrospinal fluid-

274 interstitial fluid exchange ${ }^{60}$, cerebral edema secondary to an intermittent hypoxia ${ }^{61}$ (similar to the

275 increase in brain volume and pseudoatrophy observed in multiple sclerosis), and compensatory 
276 excessive neuronal synaptic activity ${ }^{62}$ in SDB, all of which could potentially lead to an increase

277 in beta-amyloid deposition and its clearance reduction. It is therefore possible that the presence

278 of SDB is associated with $\mathrm{AD}$ risk only through beta-amyloid deposition ${ }^{42,58}$ or altered brain

279 function $^{63-65}$, but an interaction should have been observed in MCI or AD where it is generally

280 accepted that neuronal loss follows amyloid deposition. More studies are needed to better

281 understand the compensatory increase in gray matter volume in SDB suggested by several

282 studies, as well as the precise progression of brain atrophy in $\mathrm{AD}$, as both may have contributed

283 to obtaining such negative findings.

\section{BrainAGE prediction in AD and SDB}

Brain age prediction methods have been used in cognitively normal subjects ${ }^{26,66}$ and

287 several studies have used the ADNI dataset with mean absolute error (MAE) ranging from 3 to 6

288 years $^{25,27}$. We implemented an advanced sensitive BrainAGE estimation method to detect

289 pathologic brain aging, using repeated SVM models fitted on parcel-wise gray matter volume

290 data of on stratified subsamples from external cohorts, making the model less sensitive to

291 heterogeneity in images ${ }^{25}$. Compared to previous studies, while using multiple datasets for

292 training prediction model, our age prediction results were accurate with an MAE of 3.6 years in

293 HCs. Replication of previous findings in AD, taken together with acceptable MAE, is indicative

294 of reliability of our proposed method in gray matter volume assessment and age estimation

295 While there is no exact definition for accelerated brain aging, BrainAGE score has been

296 shown to be a sensitive predictor of disease progression in dementia ${ }^{27-29}$. Previous findings on

297 increased BrainAGE score in MCI and AD course ${ }^{67-69}$, are in agreement with the reported 298 accelerated aging of the demented brain shown in-vivo and ex-vivo studies ${ }^{70}$. The BrainAGE 
299 score in studies using ADNI ranged from almost zero for patients with stable MCI, to 5.7-6.2

300 years for patients with progressive MCI, and reached up to 10 years for patients with $\mathrm{AD}^{27}$. We

301 found the average 4.1 and 9 BrainAGE scores in patients with $\mathrm{AD}$ and MCI, in agreement to

302 previous findings using ADNI data. Since we did not distinguish patients with progressive from

303 stable MCI, our results in the MCI group were modest compared to other studies including

304 patients with late or progressive MCI.

\section{Conclusions}

In summary, we have confirmed the acceleration of brain atrophy and advanced brain

307 aging in MCI and AD participants from the ADNI cohort compared to healthy controls. We

308 further found that self-reported SDB in subjects with a diagnosis of HC, MCI or AD was neither

309 associated with gray matter volume reduction, nor with accelerated brain aging. While SDB is

310 suggested to propagate the aging process, amyloid burden and cognitive decline to AD, it may

311 not necessarily associate to brain atrophy and the estimated brain age in AD progession. 


\section{Acknowledgment}

314 The authors gratefully acknowledge the efforts, time, and dedication of the participants and staff

315 of the Alzheimer's Disease Neuroimaging Initiative (ADNI). Collection and sharing of data for

316 this project was funded by ADNI (National Institutes of Health Grant U01 AG024904) and DOD

317 ADNI (Department of Defense award number W81XWH-12-20012). ADNI is funded by the

318 National Institute on Aging, the National Institute of Biomedical Imaging and Bioengineering,

319 and through generous contributions from the following: AbbVie, Alzheimer's Association;

320 Alzheimer's Drug Discovery Foundation; Araclon Biotech; BioClinica, Inc.; Biogen; Bristol-

321 Myers Squibb Company; CereSpir, Inc.; Cogstate; Eisai Inc.; Elan Pharmaceuticals, Inc.; Eli

322 Lilly and Company; EuroImmun; F. Hoffmann-La Roche Ltd and its affiliated company

323 Genentech, Inc.; Fujirebio; GE Healthcare; IXICO Ltd.; Janssen Alzheimer Immunotherapy

324 Research \& Development, LLC.; Johnson \& Johnson Pharmaceutical Research \& Development

325 LLC.; Lumosity; Lundbeck; Merck \& Co., Inc.; Meso Scale Diagnostics, LLC.; NeuroRx

326 Research; Neurotrack Technologies; Novartis Pharmaceuticals Corporation; Pfizer Inc.; Piramal

327 Imaging; Servier; Takeda Pharmaceutical Company; and Transition Therapeutics. The Canadian

328 Institutes of Health Research is providing funds to support ADNI clinical sites in Canada. Private

329 sector contributions are facilitated by the Foundation for the National Institutes of Health

330 (www.fnih.org). The grantee organization is the Northern California Institute for Research and

331 Education, and the study is coordinated by the Alzheimer's Therapeutic Research Institute at the

332 University of Southern California. ADNI data are disseminated by the Laboratory for Neuro

333 Imaging at the University of Southern California. Of note, Simon B. Eickhoff is supported by the

334 Deutsche Forschungsgemeinschaft, the National Institute of Mental Health (R01-MH074457),

335 the Helmholtz Portfolio Theme "Supercomputing and Modeling for the Human Brain" and the 
336 European Union's Horizon 2020 Research and Innovation Programme under Grant Agreement

337 No. 7202070 (HBP SGA1). Ivana Rosenzweig was supported by the Wellcome Trust

$338[103952 / Z / 14 / Z]$. Ricardo Osorio's salary is supported by NIH/NIA'a R01AG056031, 339 R01AG056531 and R21AG055002. 


\section{Abbreviations:}

341 1. Magnetic resonance imaging: MRI

342 2. Sleep-disordered breathing: SDB

343 3. Obstructive Sleep Apnea: OSA

344 4. Alzheimer's disease: AD

345 5. Mild cognitive impairment: MCI

346 6. Healthy Control: HC

347 7. Apolipoprotein E4: ApoE4

348 8. Mini-Mental State Examination: MMSE

349 9. Support vector machine: SVM

350 10. Voxel-based morphometry: VBM

351 11. Computational Anatomy Toolbox: CAT

352 12. Statistical Parametric Mapping: SPM

353 13. Family-wise error: FWE

354 14. Analysis of variance: ANOVA

\section{Disclosure statement}

357 Authors have no financial or non-financial conflict of interest to disclose.

\section{Study funding}

359 This research did not receive any specific grant from funding agencies in the public, commercial,

360 or not-for-profit sectors. ADNI study has been founded by National Institutes of Health Grant

361 U01 AG024904 and DOD ADNI, Department of Defense award number W81XWH-12-20012. 
362 Simon B. Eickhoff is supported by the Deutsche Forschungsgemeinschaft, the National Institute 363 of Mental Health (R01-MH074457), the Helmholtz Portfolio Theme "Supercomputing and 364 Modeling for the Human Brain" and the European Union's Horizon 2020 Research and 365 Innovation Programme under Grant Agreement No. 7202070 (HBP SGA1). Ivana Rosenzweig 366 was supported by the Wellcome Trust [103952/Z/14/Z].Ricardo Osorio's salary is supported by 367 NIH/NIA'a R01 AG056031, R01AG056531 and R21 AG055002. 


\section{References:}

369 1. Nichols E, Szoeke CEI, Vollset SE, et al. Global, regional, and national burden of Alzheimer's disease and 370 other dementias, 1990-2016: a systematic analysis for the Global Burden of Disease Study 2016. Lancet Neurol. 2019;18(1):88-106. doi:10.1016/S1474-4422(18)30403-4

372 2. $\mathrm{Xu} \mathrm{W}$, Tan L, Wang H-F, et al. Meta-analysis of modifiable risk factors for Alzheimer's disease. J Neurol 373 Neurosurg Psychiatry. 2015;86(12):1299-1306. doi:10.1136/JNNP-2015-310548

374 3. Alford S, Patel D, Perakakis N, Mantzoros CS. Obesity as a risk factor for Alzheimer's disease: weighing 375 the evidence. Obes Rev. 2018;19(2):269-280. doi:10.1111/obr.12629

376 4. Emamian F, Khazaie H, Tahmasian M, et al. The association between obstructive sleep apnea and Alzheimer's disease: A meta-analysis perspective. Front Aging Neurosci. 2016;8(APR):1-8. doi:10.3389/fnagi.2016.00078

5. Adult Obstructive Sleep Apnea Task Force of the American Academy of Sleep Medicine, Epstein LJ, Kristo D, et al. Clinical Guidline for the Evaluation, Management, and Long-term Care of Obstructive Sleep Apnea in Adults. J Clin Sleep Med. 2009;5(9):263-276.

6. Yaffe K, Laffan AM, Harrison SL, et al. Sleep-Disordered Breathing, Hypoxia, and Risk of Mild Cognitive Impairment and Dementia in Older Women. JAMA. 2011;306(6):486-495. doi:10.1001/jama.2011.1115

7. Osorio RS, Gumb T, Pirraglia E, et al. Sleep-disordered breathing advances cognitive decline in the elderly. Neurology. 2015:1-8. doi:10.1212/WNL.0000000000001566

386 8. Karas GB, Scheltens P, Rombouts SARB, et al. Global and local gray matter loss in mild cognitive impairment and Alzheimer's disease. Neuroimage. 2004;23(2):708-716.

9. Jack CR, Shiung MM, Gunter JL, et al. Comparison of different MRI brain atrophy rate measures with clinical disease progression in AD. Neurology. 2004;62(4):591-600.

392 10. Fox NC, Schott JM. Imaging cerebral atrophy: normal ageing to Alzheimer's disease. Lancet. 2004;363(9406):392-394. doi:10.1016/S0140-6736(04)15441-X

394 11. Pasquini L, Rahmani F, Maleki-Balajoo S, et al. Medial Temporal Lobe Disconnection and 
doi:10.3233/ADR-190121

397 12. Good CD, Johnsrude IS, Ashburner J, Henson RNA, Friston KJ, Frackowiak RSJ. A Voxel-Based

398 Morphometric Study of Ageing in 465 Normal Adult Human Brains. Neuroimage. 2001;14(1):21-36. doi:10.1006/NIMG.2001.0786

400 13. Weng H-H, Tsai Y-HY-H, Chen C-F, et al. Mapping gray matter reductions in obstructive sleep apnea: an 401 activation likelihood estimation meta-analysis. Sleep. 2014;37(1):167-175. doi:10.5665/sleep.3330

402 14. Celle S, Delon-Martin C, Roche F, Barthelemy J-C, Pepin J-L, Dojat M. Desperately seeking grey matter volume changes in sleep apnea: A methodological review of magnetic resonance brain voxel-based morphometry studies. Sleep Med Rev. 2016;25:112-120. doi:10.1016/j.smrv.2015.03.001

15. Torelli F, Moscufo N, Garreffa G, et al. Cognitive profile and brain morphological changes in obstructive

407 16. Joo EY, Jeon S, Kim ST, Lee J-M, Hong SB. Localized cortical thinning in patients with obstructive sleep

17. Yun C-H, Lee H-Y, Lee SK, et al. Amyloid Burden in Obstructive Sleep Apnea. J Alzheimer's Dis.

$410 \quad$ 2017;59(1):21-29. doi:10.3233/JAD-161047

411 18. Fortea J, Vilaplana E, Alcolea D, et al. Cerebrospinal fluid $\beta$-amyloid and phospho-tau biomarker

412 interactions affecting brain structure in preclinical Alzheimer disease. Ann Neurol. 2014;76(2):223-230.

413 doi:10.1002/ana.24186

414 19. Fatouleh RH, Hammam E, Lundblad LC, et al. Functional and structural changes in the brain associated 415 with the increase in muscle sympathetic nerve activity in obstructive sleep apnoea. NeuroImage Clin. 2014;6:275-283. doi:10.1016/J.NICL.2014.08.021

417 20. Lundblad LC, Fatouleh RH, Hammam E, McKenzie DK, Macefield VG, Henderson LA. Brainstem changes $418 \quad$ associated with increased muscle sympathetic drive in obstructive sleep apnoea. Neuroimage. 2014;103:258266. doi:10.1016/J.NEUROIMAGE.2014.09.031

420 21. Kumar R, Farahvar S, Ogren JA, et al. Brain putamen volume changes in newly-diagnosed patients with 421 obstructive sleep apnea. NeuroImage Clin. 2014;4:383-391. doi:10.1016/J.NICL.2014.01.009

422 22. Rosenzweig I, Kempton MJ, Crum WR, et al. Hippocampal Hypertrophy and Sleep Apnea: A Role for the 423 Ischemic Preconditioning? Annunziato L, ed. PLoS One. 2013;8(12):e83173. 
425 23. Baril A-AA-A, Gagnon K, Brayet P, et al. Gray Matter Hypertrophy and Thickening with Obstructive Sleep

426 Apnea in Middle-aged and Older Adults. Am J Respir Crit Care Med. 2017;195(11):1509-1518.

427 doi:10.1164/rccm.201606-12710C

428 24. Lin W-C, Huang C-C, Chen H-L, et al. Longitudinal brain structural alterations and systemic inflammation

429 in obstructive sleep apnea before and after surgical treatment. J Transl Med. 2016;14(1):139.

$430 \quad$ doi: $10.1186 / s 12967-016-0887-8$

431 25. Varikuti DP, Genon S, Sotiras A, et al. Evaluation of non-negative matrix factorization of grey matter in age 432 prediction. Neuroimage. 2018;173:394-410. doi:10.1016/J.NEUROIMAGE.2018.03.007

433 26. Franke K, Luders E, May A, Wilke M, Gaser C. Brain maturation: Predicting individual BrainAGE in children and adolescents using structural MRI. Neuroimage. 2012;63(3):1305-1312. doi:10.1016/J.NEUROIMAGE.2012.08.001

436 27. Cole JH, Marioni RE, Harris SE, Deary IJ. Brain age and other bodily 'ages': implications for neuropsychiatry. Mol Psychiatry. 2019;24(2):266-281. doi:10.1038/s41380-018-0098-1

438 28. Löwe LC, Gaser C, Franke K, Initiative for the ADN. The Effect of the APOE Genotype on Individual 439 BrainAGE in Normal Aging, Mild Cognitive Impairment, and Alzheimer's Disease. Ginsberg SD, ed. PLoS One. 2016;11(7):e0157514. doi:10.1371/journal.pone.0157514

441 29. Gaser C, Franke K, Klöppel S, Koutsouleris N, Sauer H, Initiative ADN. BrainAGE in Mild Cognitive 442 Impaired Patients: Predicting the Conversion to Alzheimer's Disease. Ginsberg SD, ed. PLoS One. 443 2013;8(6):e67346. doi:10.1371/journal.pone.0067346

444 30. Petersen RC, Aisen PS, Beckett LA, et al. Alzheimer's Disease Neuroimaging Initiative (ADNI): Clinical 445 characterization. Neurology. 2010;74(3):201-209. doi:10.1212/WNL.0b013e3181cb3e25

446 31. Jack CR, Bernstein MA, Fox NC, et al. The Alzheimer's disease neuroimaging initiative (ADNI): MRI methods. J Magn Reson Imaging. 2008;27(4):685-691. doi:10.1002/jmri.21049

448 32. C. Gaser, R. Dahnke. CAT - a computational anatomy toolbox for the analysis of structural MRI data. OHBM 2016 - 22nd Annu Meet Organ Hum Brain Mapping6. 2016;2016:336-348.

450 33. Schaefer A, Kong R, Gordon EM, et al. Local-Global Parcellation of the Human Cerebral Cortex from 451 Intrinsic Functional Connectivity MRI. Cereb Cortex. 2018;28(9):3095-3114. doi:10.1093/cercor/bhx179 
452 34. Fan L, Li H, Zhuo J, et al. The Human Brainnetome Atlas: A New Brain Atlas Based on Connectional Architecture. Cereb Cortex. 2016;26(8):3508-3526. doi:10.1093/cercor/bhw157

$454 \quad 35 . \quad$ Bludau S, Mühleisen TW, Eickhoff SB, Hawrylycz MJ, Cichon S, Amunts K. Integration of transcriptomic and cytoarchitectonic data implicates a role for MAOA and TAC1 in the limbic-cortical network. Brain Struct Funct. 2018;223(5):2335-2342. doi:10.1007/s00429-018-1620-6

457 36. Caspers S, Moebus S, Lux S, et al. Studying variability in human brain aging in a population-based German cohort-rationale and design of 1000BRAINS. Front Aging Neurosci. 2014;6(JUL):149. doi:10.3389/fnagi.2014.00149

37. Shafto MA, Tyler LK, Dixon M, et al. The Cambridge Centre for Ageing and Neuroscience (Cam-CAN) study protocol: a cross-sectional, lifespan, multidisciplinary examination of healthy cognitive ageing. $B M C$ Neurol. 2014;14:204. doi:10.1186/s12883-014-0204-1

39. Zuo X-N, Anderson JS, Bellec P, et al. An open science resource for establishing reliability and

40. Nooner KB, Colcombe SJ, Tobe RH, et al. The NKI-Rockland Sample: A Model for Accelerating the Pace of Discovery Science in Psychiatry. Front Neurosci. 2012;6:152. doi:10.3389/fnins.2012.00152 reproducibility in functional connectomics. Sci Data. 2014;1(1):140049. doi:10.1038/sdata.2014.49

472 42. Bubu OM, Pirraglia E, Andrade AG, et al. Obstructive Sleep Apnea and Longitudinal Alzheimer's disease 473 biomarker changes. Sleep. February 2019. doi:10.1093/sleep/zsz048

474 43. Yang J, Pan P, Song W, et al. Voxelwise meta-analysis of gray matter anomalies in Alzheimer's disease and mild cognitive impairment using anatomic likelihood estimation. J Neurol Sci. 2012;316(1-2):21-29.

477 44. Wang W-Y, Yu J-T, Liu Y, et al. Voxel-based meta-analysis of grey matter changes in Alzheimer's disease. Transl Neurodegener. 2015;4(1):6. doi:10.1186/s40035-015-0027-z

479 45. Nickl-Jockschat T, Kleiman A, Schulz JB, et al. Neuroanatomic changes and their association with cognitive 
decline in mild cognitive impairment: a meta-analysis. Brain Struct Funct. 2012;217(1):115-125. doi:10.1007/s00429-011-0333-x

482 46. Joo EY, Tae WS, Lee MJ, et al. Reduced brain gray matter concentration in patients with obstructive sleep 483 apnea syndrome. Sleep. 2010;33(2):235-241. doi:10.1093/sleep/33.2.235

484 47. Taylor KS, Millar PJ, Murai H, et al. Cortical autonomic network gray matter and sympathetic nerve activity 485 in obstructive sleep apnea. Sleep. 2018;41(2). doi:10.1093/sleep/zsx208

486 48. Morrell MJ, Jackson ML, Twigg GL, et al. Changes in brain morphology in patients with obstructive sleep apnoea. Thorax. 2010;65(10):908-914. doi:10.1136/thx.2009.126730

488 49. O'Donoghue FJ, Briellmann RS, Rochford PD, et al. Cerebral Structural Changes in Severe Obstructive Sleep Apnea. Am J Respir Crit Care Med. 2005;171(10):1185-1190. doi:10.1164/rccm.200406-738OC

490 50. Shi Y, Chen L, Chen T, et al. A Meta-analysis of Voxel-based Brain Morphometry Studies in Obstructive 491 Sleep Apnea. Sci Rep. 2017;7(1):10095. doi:10.1038/s41598-017-09319-6

492 51. Tahmasian M, Rosenzweig I, Eickhoff SBSB, et al. Structural and functional neural adaptations in obstructive sleep apnea: an activation likelihood estimation meta-analysis. Neurosci Biobehav Rev. 2016;65:142-156. doi:10.1016/j.neubiorev.2016.03.026

495 52. Lutsey PL, Norby FL, Gottesman RF, et al. Sleep Apnea, Sleep Duration and Brain MRI Markers of Cerebral Vascular Disease and Alzheimer's Disease: The Atherosclerosis Risk in Communities Study (ARIC). Yung W, ed. PLoS One. 2016;11(7):e0158758. doi:10.1371/journal.pone.0158758

498 53. Cross NE, Memarian N, Duffy SL, et al. Structural brain correlates of obstructive sleep apnoea in older 499 adults at risk for dementia. Eur Respir J. 2018;52(1). doi:10.1183/13993003.00740-2018

500 54. Celle S, Peyron R, Faillenot I, et al. Undiagnosed sleep-related breathing disorders are associated with focal 501 brainstem atrophy in the elderly. Hum Brain Mapp. 2009;30(7):2090-2097. doi:10.1002/hbm.20650

502 55. Canessa N, Castronovo V, Cappa SF, et al. Sleep apnea: Altered brain connectivity underlying a working503 memory challenge. NeuroImage Clin. 2018;19:56-65. doi:10.1016/j.nicl.2018.03.036

504 56. Liguori C, Mercuri NB, Izzi F, et al. Obstructive Sleep Apnea is Associated With Early but Possibly 505 Modifiable Alzheimer's Disease Biomarkers Changes. Sleep. 2017;40(5). doi:10.1093/sleep/zsx011 506 57. Spira AP, Gamaldo AA, An Y, et al. Self-reported Sleep and $\beta$-Amyloid Deposition in Community507 Dwelling Older Adults. JAMA Neurol. 2013;70(12):1537-1543. doi:10.1001/jamaneurol.2013.4258 
58. Sharma RA, Varga AW, Bubu OM, et al. Obstructive Sleep Apnea Severity Affects Amyloid Burden in Cognitively Normal Elderly. A Longitudinal Study. Am J Respir Crit Care Med. 2018;197(7):933-943. doi:10.1164/rccm.201704-0704OC

511 59. Kallenberg K, Bailey DM, Christ S, et al. Magnetic Resonance Imaging Evidence of Cytotoxic Cerebral Edema in Acute Mountain Sickness. J Cereb Blood Flow Metab. 2007;27(5):1064-1071. doi:10.1038/sj.jcbfm.9600404

514 60. Ju Y-ES, Finn MB, Sutphen CL, et al. Obstructive sleep apnea decreases central nervous system-derived proteins in the cerebrospinal fluid. Ann Neurol. 2016;80(1):154-159. doi:10.1002/ana.24672

516 61. Spira AP, An Y, Wu MN, et al. Excessive daytime sleepiness and napping in cognitively normal adults: 517 associations with subsequent amyloid deposition measured by PiB PET. Sleep. 2018;41(10). doi:10.1093/sleep/zsy152

519 62. Polsek D, Gildeh N, Cash D, et al. Obstructive sleep apnoea and Alzheimer's disease: In search of shared pathomechanisms. Neurosci Biobehav Rev. 2018;86(June 2017):142-149.

522 63. Thomas RJ, Rosen BR, Stern CE, Weiss JW, Kwong KK. Functional imaging of working memory in 523 obstructive sleep-disordered breathing. J Appl Physiol. 2005;98(6):2226-2234. doi:10.1152/japplphysiol.01225.2004

525 64. Park B, Palomares JA, Woo MA, et al. Disrupted functional brain network organization in patients with 526 obstructive sleep apnea. Brain Behav. 2016;6(3):e00441. doi:10.1002/brb3.441

527 65. Chen L, Fan X, Li H, et al. Topological Reorganization of the Default Mode Network in Severe Male 528 Obstructive Sleep Apnea. Front Neurol. 2018;9:363. doi:10.3389/fneur.2018.00363

529 66. Aycheh HM, Seong J-K, Shin J-H, et al. Biological Brain Age Prediction Using Cortical Thickness Data: A 530 Large Scale Cohort Study. Front Aging Neurosci. 2018;10:252. doi:10.3389/fnagi.2018.00252

531 67. Liem F, Varoquaux G, Kynast J, et al. Predicting brain-age from multimodal imaging data captures 532 cognitive impairment. Neuroimage. 2017;148:179-188. doi:10.1016/J.NEUROIMAGE.2016.11.005

533 68. Caballero MÁA, Klöppel S, Dichgans M, Ewers M. Spatial patterns of longitudinal gray matter change as predictors of concurrent cognitive decline in amyloid positive healthy subjects. Marchant N, ed. $J$

535 Alzheimer's Dis. 2016;55(1):343-358. doi:10.3233/JAD-160327 
536 69. Beheshti I, Maikusa N, Matsuda H. The association between "Brain-Age Score" (BAS) and traditional

537 neuropsychological screening tools in Alzheimer's disease. Brain Behav. 2018;8(8):e01020.

538 doi:10.1002/brb3.1020

539 70. Mecocci P, Boccardi V, Cecchetti R, et al. A Long Journey into Aging, Brain Aging, and Alzheimer's

540 Disease Following the Oxidative Stress Tracks. J Alzheimers Dis. 2018;62(3):1319-1335. doi:10.3233/JAD-

541

542 71. Gaspar LS, Álvaro AR, Moita J, Cavadas C. Obstructive Sleep Apnea and Hallmarks of Aging. Trends Mol 543 Med. 2017;23(8):675-692. doi:10.1016/j.molmed.2017.06.006

544 72. Gozal D. The ageing brain in sleep apnoea: paradoxical resilience, survival of the fittest, or simply

$545 \quad$ comparing apples and oranges? Eur Respir J. 2018;51(6):1800802. doi:10.1183/13993003.00802-2018

546 73. Scheller E, Schumacher L V., Peter J, et al. Brain Aging and APOE $\varepsilon 4$ Interact to Reveal Potential Neuronal

547 Compensation in Healthy Older Adults. Front Aging Neurosci. 2018;10:74. doi:10.3389/fnagi.2018.00074 
Table 1. Characteristics of the study subjects

\begin{tabular}{llll}
\hline & SDB- & SDB+ & P value \\
\cline { 2 - 4 } & N: 165 & n:165 & \\
\hline Age (Mean (SD)) & $73.99(7.70)$ & $74.91(7.18)$ & 0.26 \\
Sex, Female (\%) & $61(37.0)$ & $48(29.1)$ & 0.16 \\
Cognitive status (\%) & & & 1.00 \\
$\quad$ AD & $24(14.5)$ & $24(14.5)$ & \\
MCI & $111(67.3)$ & $111(67.3)$ & \\
HC & $30(18.2)$ & $30(18.2)$ & \\
BMI (Mean (SD)) & $28.97(5.95)$ & $29.08(5.45)$ & 0.86 \\
Education years $\quad(M e a n$ & $16.07(2.75)$ & $16.16(2.65)$ & 0.74 \\
(SD)) & $18(10.9)$ & $18(10.9)$ & 1.00 \\
Handedness = Left (\%) & & & 0.13 \\
Apoe4 allele count (\%) & $71(46.7)$ & $94(58.0)$ & \\
0 & $64(42.1)$ & $53(32.7)$ & \\
1 & $17(11.2)$ & $15(9.3)$ & \\
2 & $26.07(4.13)$ & $25.44(4.93)$ & 0.25 \\
MMSE (Mean (SD))* & $0(0.0)$ & $56(33.9)$ & - \\
CPAP/Surgery (\%)* & $118(71.5)$ & $124(75.2)$ & 0.53 \\
Protocol, MP-RAGE (\%) & &
\end{tabular}

SDB: Sleep-disordered breathing, AD: Alzheimer's disease, MCI: Mild cognitive impairment, HC: healthy control, BMI: Body-mass index, MMSE: Mini-mental state examination, MPRAGE: 3D magnetization prepared rapid gradient echo, CPAP: Continuous positive airway pressure

*Not included in the matching 


\section{Figures' legends}

Figure 1. Main processing steps for parcel based volumetric study and age prediction based on gray matter morphometry. A1. T1 brain images of 2089 non-demented age, sex, and site stratified subjects were acquired through several imaging databases for development of ageprediction model (Training images). To obtain voxel-based gray matter volume data, standard pre-processing steps including normalization, segmentation and modulation for non-linear transformations have been done using Computational Anatomical Toolbox 12 (CAT12). A biologically informed compression of the voxel-wise gray matter volume data to 600 cortical and 37 subcortical regions was applied accordingly. B. Parcel-based results were then used as input for training the support vector machine (SVM) used for age-prediction model.

A2. Similar pre-processing steps were done on T1 brain images of study-specific SDB+ and SDB- subjects (Study-specific images). Parcel-based results were used in two parallel analyses;

1) C. inputted to partial ANOVA tests for gray matter volume assessment according to presence of SDB and cognitive status as contrasts and 2) D. Decomposed with an OPNMF approach and inputted in the age prediction SVM model developed on the training images.

Figure 2. Association between volumetric data of cortical and subcortical parcels and age and cognitive status of subjects. Gray matter volume differences in 600 cortical parcels and 37 subcortical volume was assessed using three steps of using $\mathrm{F}$ value of an n-way analysis of variance as reference statistics, running 10,000 permutations per randomly shuffling different parcels, under assumption of label exchangeability, and correction of $\mathrm{p}$ values using family wise error (FWE) method. Significant parcels are illustrated as the heated areas on the brain maps considering (A) age and (B) cognitive status. Since there were no significant results regarding SDB presence or SDB-by-diagnosis interaction, results according these factors have not been illustrated here.

Figure 3. Results of the BrainAGE prediction method based on the presence of SDB and cognitive status. A. Relationship between chronological age and the predicted age from T1 images in the AD, MCI and HC groups. There is an evident higher predicted age for the participants $\mathrm{AD}$ and $\mathrm{MCI}$ compared to $\mathrm{HC}$ group, in accordance with advanced pathological 
brain aging in the $\mathrm{AD}$ course. $\mathrm{B}$. The BrainAGE score shows positive and bigger deviation from chronological age in the AD and MCI groups. C. Despite the significantly higher BrainAGE deviation associated with $\mathrm{AD}$ and $\mathrm{MCI}$, no significant deviation was seen between BrainAGE score of SDB subgroups. 


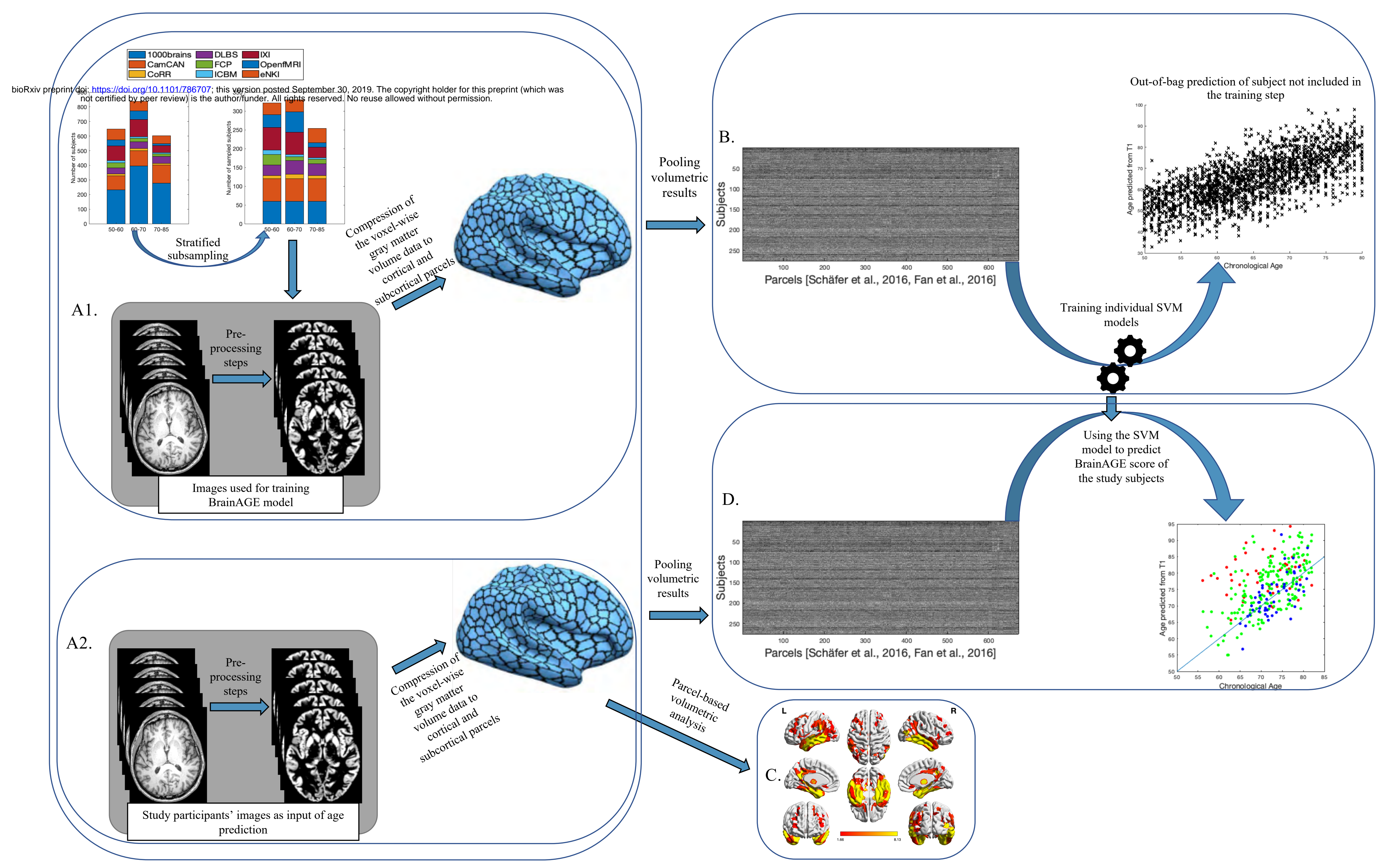




\section{$\boldsymbol{x}$}

bioRxiv preprint doi: https /doi.org/10.1201786707; this version posted September 30 2019. The copyright holder for this preprint (which was not certified by peer re
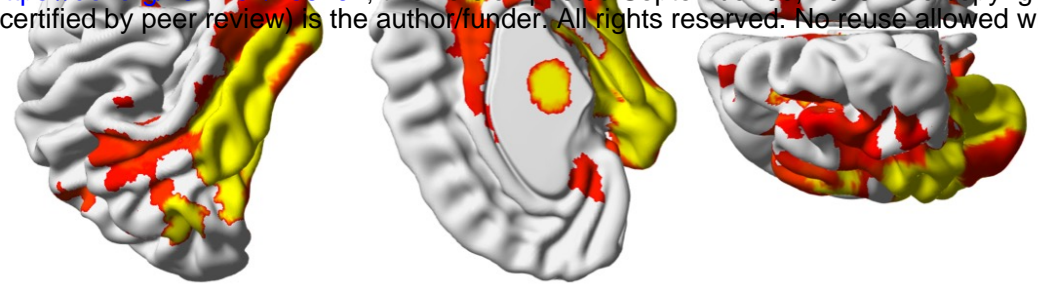

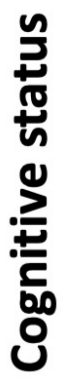
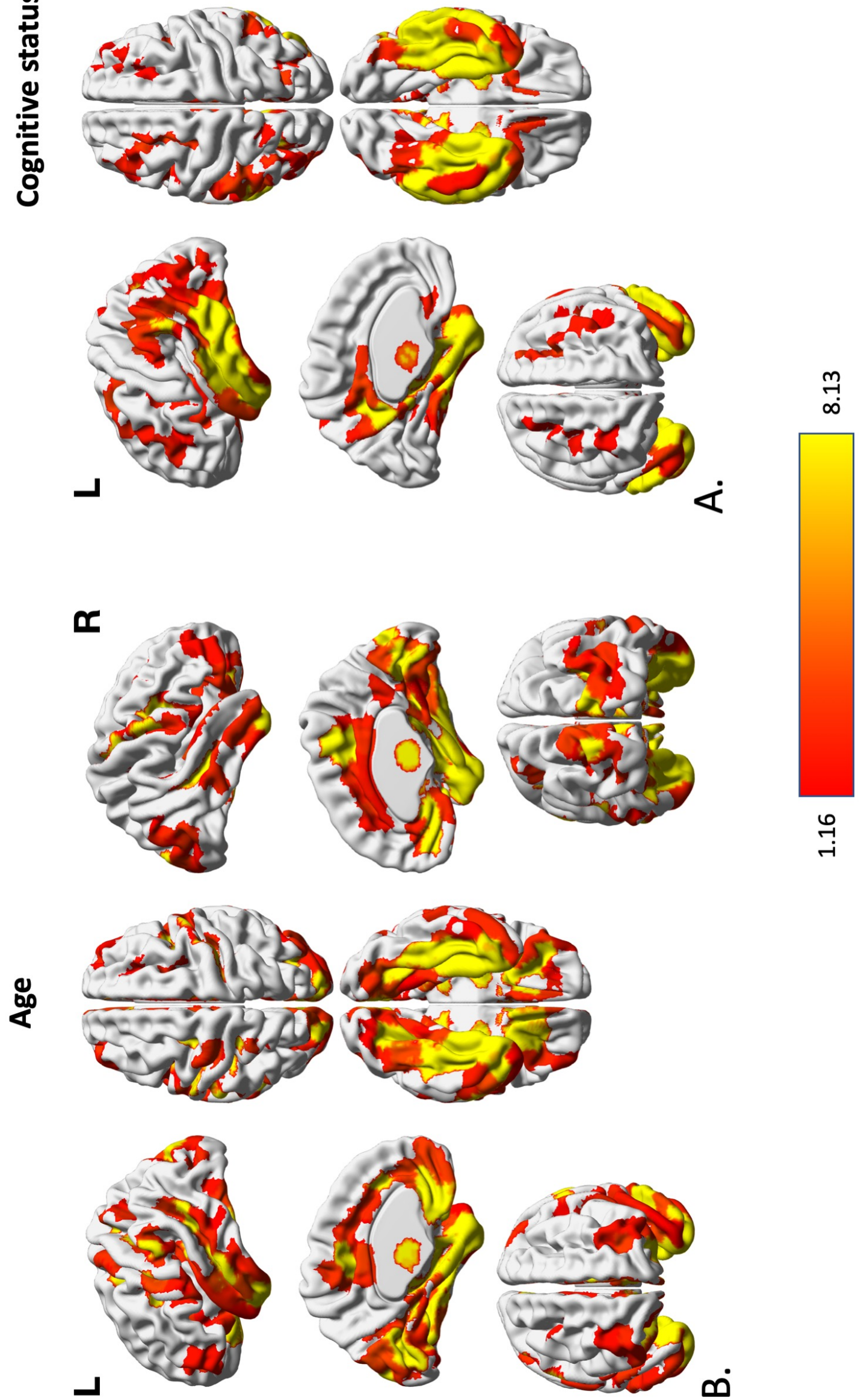

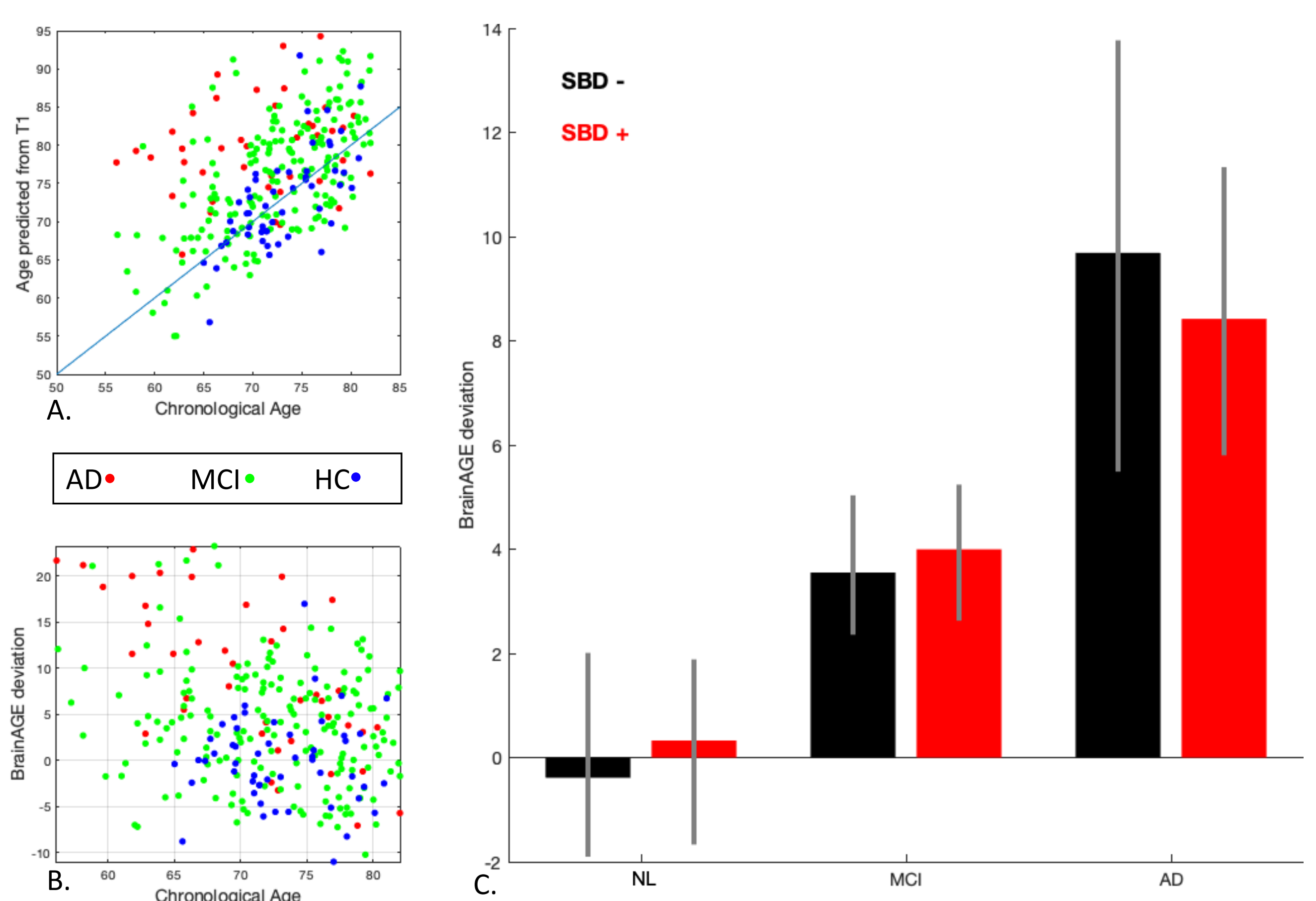